\title{
Genetic Diversity Studies in Sesamum
}

\author{
Rohini Y. Patil*, K. K. Barhate, B. A. Tagad and V. S. Girase \\ Botany Section, College of Agriculture, (M. S.), Dhule, India \\ *Corresponding author
}

\begin{tabular}{|l|}
\hline Key w o r d s \\
Genetic diversity, \\
Cluster distance, \\
Sesame
\end{tabular}

\author{
A B S T R A C T
}

Genetic Diversity Studies in Sesamum was carried for forty-two genotypes to study the nature and extent of genetic diversity for the characters viz; days to $50 \%$ flowering, days to maturity, plant height at maturity $(\mathrm{cm})$, number of branches per plant, number of capsules per plant, number of seeds per capsule, 1000 seed weight $(\mathrm{g})$, oil content $(\%)$ and seed yield per plant $(\mathrm{g})$. Analysis of variance revealed significant difference among the genotypes for all the characters. Based on $\mathrm{D}^{2}$ value, the genotypes were grouped into nine clusters indicating wider genetic diversity in the germplasm collections of Sesamum from different geographical origin. Characters, plant height at maturity (48.03\%) contributed more in genetic divergence. Which was followed by number of capsules per plant (13.82 $\%$ ), oil content $(10.69 \%)$, days to $50 \%$ flowering $(9.06 \%)$, seed yield per plant (5.87) and number of branches per plant $(5.57 \%)$ as main characters contributing to the genetic divergence in present material. The clustering pattern suggested that the genotypes of the same origin were distributed into different clusters indicating the absence of parallelism between clustering and geographical distribution. These genotypes were distinct and diverse and can be classified as promising genotypes. Inter-crossing among them would lead to upgrade base in the base population and opportunities for obtaining the high heterotic effect and also to recover desirable transgressive segregents and wide spectrum of variability in subsequent generations. Maximum inter-cluster distance was noticed between cluster VII and VIII $\left(\mathrm{D}^{2}=9.71\right)$.

\section{Introduction}

Sesame (Sesamum indicum L.) is probably the most ancient oilseed known and used by man its domestication is lost in the midst of antiquity The genus Sesamum belongs to the order tubiflorea, family pedaliaceae which consist of sixteen genera and sixty species, but only Sesamum indicum $(2 \mathrm{n}=26)$ has been recognized as cultivated species Sesamum indicum L. which is known variously as sesamum, til, gingelly, sissim, gergelim etc. Sesamum is a self-pollinated crop.

Sesame seed is rich in oil, protein, carbohydrate, fibre and minerals. Sesamum is an important source of edible oil and is widely used in food products especially in bakery foods and animal feed. Sesamum seed contains 40-64 $\%$ oil and $25 \%$ protein with antioxidants lignins such as sesamolin and sesamin. Sesamum is better known as "Queen of Oilseeds". 
Total area of sesamum in India is 19.51 lakh ha and production 8.50 lakh tons and productivity $436(\mathrm{~kg} / \mathrm{ha})$. In Maharashtra, it is grown on area of 0.17 lakh ha with production of 0.038 lakh tons and productivity of 223 $\mathrm{kg} / \mathrm{ha}$.

The genetic diversity is a crucial factor in determining the success of hybridization programme and its important in crop improvement has long been recognized by breeder. The more diverse parents within overall limit of fitness, the greater are the chances of heterotic $F_{1}$ 's and broad spectrum of variability in segregating generation (Arunachalam, 1981; Falconer, 1989). Therefore, the first step in any crop breeding programme is to access genetic variability. Yield and yield contributing characters are controlled by polygene and highly influenced by environment; the exploration of genetic variability in available germplasm is prerequisite. Therefore, evaluation of germplasm to local conditions is very important.

Genetic diversity is widely accepted that information about germplasm diversity and genetic relatedness among elite breeding material is a fundamental element in plant breeding. Genetic diversity is very important factor for any hybridization programme aiming at genetic improvement of yield especially in self-pollinated crops. Different methods have been used to assess genetic diversity, of which Mahalanobis's (1936) $\mathrm{D}^{2}$ statistic is the most efficient tool for estimating genetic divergence. Genetic diversity plays important role, because hybrids between closely related parents.

\section{Materials and Methods}

The experimental material comprising forty two genotypes of sesame were grown in Randomized Block Design with three replications at the research farm of Botany, College of Agriculture, Dhule, during Kharif season of 2019. Each entry was represented by single row of 4.5 meter length with spacing of $30 \mathrm{~cm}$ between the rows and $15 \mathrm{~cm}$ between the plants. Data were recorded on five randomly and competitive plants of each genotype from each replication for nine characters viz., days to $50 \%$ flowering, days to maturity, plant height at maturity $(\mathrm{cm})$, number of branches per plant, number of capsules per plant, number of seeds per capsule, 1000 seed weight (g), oil content (\%) and seed yield per plant $(\mathrm{g})$. The mean of five plants was subjected to statistical analysis. The data for different characters were statistically analyzed for significance by using analysis of variance technique described by Panse and Sukhatme (1995). The analysis of divergence was carried out by $\mathrm{D}^{2}$ statistic of Mahalnobis (1936) as described by Rao (1952). All $\mathrm{D}^{2}$ values are arranged in matrix form, based on the degree of divergence $\left(D^{2}\right.$ values) between any two genotypes. A logical grouping of genotypes was done following Tocher's method (Rao, 1952). The possible limits to parental divergence within which there were reasonably high chances for occurrence of heterosis were calculated following Arunachalam and Bandopadhyaya (1984). They advised to delineate the divergence among parents into four divergence classes (DC).

\section{Results and Discussion}

Plant breeding deals with the management of genetic variability. Hence, the presence of genetic variability in the available germplasm of a crop is of immense value to design a selection procedure and to identify the superior genotypes. It is, therefore, necessary to classify and utilize this variability systematically for genetic up-gradation of biological population (Table 1-9). 
Table.1 Mean performance of forty-two genotypes for nine different characters in Sesamum

\begin{tabular}{|c|c|c|c|c|c|c|c|c|c|c|}
\hline Sr.No. & Genotypes & $\begin{array}{c}\text { Days to } \\
50 \% \\
\text { flowering }\end{array}$ & $\begin{array}{l}\text { Days to } \\
\text { maturity }\end{array}$ & $\begin{array}{c}\text { Plant } \\
\text { height at } \\
\text { maturity } \\
\text { (cm) }\end{array}$ & $\begin{array}{c}\text { No. of } \\
\text { branches/ } \\
\text { plant }\end{array}$ & $\begin{array}{c}\text { No. of } \\
\text { capsules/plant }\end{array}$ & $\begin{array}{c}\text { No. of } \\
\text { seeds/capsule }\end{array}$ & $\begin{array}{l}1000 \text { seed } \\
\text { weight }(g)\end{array}$ & $\begin{array}{c}\text { Seed } \\
\text { yield/plant } \\
(\mathrm{g})\end{array}$ & $\begin{array}{l}\text { Oil content } \\
(\%)\end{array}$ \\
\hline 1 & DHS-1 & 47.33 & 103.33 & 84.33 & 2.23 & 36.23 & 43.46 & 3.06 & 5.93 & 49.16 \\
\hline 2 & DHS-2 & 45.33 & 103.66 & 94.86 & 2.43 & 52.36 & 47.86 & 3.20 & 7.40 & 47.76 \\
\hline 3 & DHS-3 & 43.66 & 102.66 & 102.46 & 3.10 & 35.23 & 52.43 & 3.06 & 6.49 & 48.66 \\
\hline 4 & DHS-4 & 46.00 & 102.33 & 118.30 & 3.16 & 47.63 & 47.23 & 3.43 & 8.48 & 44.33 \\
\hline 5 & DHS-5 & 46.66 & 103.00 & 151.66 & 3.20 & 57.43 & 52.70 & 2.96 & 9.92 & 46.80 \\
\hline 6 & DHS-6 & 47.00 & 104.00 & 167.16 & 2.56 & 32.53 & 50.23 & 3.16 & 6.61 & 44.13 \\
\hline 7 & DHS-7 & 44.33 & 104.00 & 90.20 & 1.96 & 34.96 & 47.93 & 2.83 & 5.79 & 45.40 \\
\hline 8 & DHS-8 & 44.33 & 103.33 & 123.86 & 3.06 & 47.36 & 45.73 & 2.93 & 6.55 & 50.16 \\
\hline 9 & DHS-9 & 45.66 & 103.00 & 107.53 & 2.63 & 44.16 & 50.36 & 3.13 & 8.05 & 51.86 \\
\hline 10 & DHS-10 & 47.33 & 102.66 & 84.46 & 2.00 & 23.63 & 44.30 & 3.20 & 6.05 & 44.30 \\
\hline 11 & DHS-11 & 42.33 & 101.66 & 69.66 & 2.73 & 47.70 & 43.26 & 2.96 & 7.71 & 52.36 \\
\hline 12 & DHS-12 & 45.33 & 103.33 & 74.16 & 2.00 & 33.43 & 48.73 & 2.63 & 5.54 & 46.00 \\
\hline 13 & DHS-13 & 47.33 & 101.66 & 84.00 & 2.50 & 41.70 & 46.93 & 2.66 & 6.87 & 46.33 \\
\hline 14 & DHS-14 & 47.33 & 101.66 & 89.66 & 2.26 & 34.63 & 49.23 & 3.10 & 6.32 & 33.46 \\
\hline 15 & DHS-15 & 41.66 & 103.00 & 85.33 & 2.16 & 36.46 & 47.30 & 2.83 & 6.28 & 49.70 \\
\hline 16 & DHS-16 & 45.00 & 102.00 & 164.93 & 2.70 & 46.76 & 51.03 & 3.06 & 8.25 & 46.60 \\
\hline 17 & DHS-17 & 40.66 & 101.00 & 83.50 & 2.00 & 36.96 & 48.60 & 2.73 & 8.20 & 47.63 \\
\hline 18 & DHS-18 & 42.00 & 102.33 & 101.33 & 2.83 & 55.86 & 50.40 & 3.10 & 9.59 & 49.00 \\
\hline 19 & DHS-19 & 41.00 & 103.33 & 149.80 & 2.83 & 48.96 & 52.03 & 2.80 & 8.30 & 45.36 \\
\hline
\end{tabular}


Table.1 Contd...

\begin{tabular}{|c|c|c|c|c|c|c|c|c|c|c|}
\hline 20 & DHS-20 & 41.00 & 103.33 & 168.70 & 2.46 & 36.60 & 48.40 & 2.66 & 6.42 & 45.30 \\
\hline 21 & DHS-21 & 44.33 & 105.33 & 103.93 & 2.60 & 42.43 & 45.00 & 3.20 & 7.08 & 49.56 \\
\hline 22 & DHS-22 & 47.66 & 108.00 & 94.93 & 2.03 & 38.60 & 53.00 & 2.70 & 7.43 & 51.46 \\
\hline 23 & DHS-23 & 47.00 & 108.66 & 118.00 & 3.16 & 55.40 & 54.06 & 2.80 & 9.09 & 48.96 \\
\hline 24 & DHS-24 & 47.66 & 102.33 & 90.20 & 2.73 & 48.00 & 51.23 & 2.90 & 8.24 & 45.90 \\
\hline 25 & DHS-25 & 47.00 & 105.00 & 63.60 & 2.36 & 37.20 & 48.66 & 2.90 & 6.49 & 45.20 \\
\hline 26 & DHS-26 & 47.33 & 106.33 & 107.76 & 2.03 & 47.46 & 45.43 & 3.13 & 7.64 & 51.73 \\
\hline 27 & DHS-27 & 46.66 & 107.33 & 110.33 & 3.16 & 46.46 & 51.03 & 3.23 & 8.64 & 52.43 \\
\hline 28 & DHS-28 & 46.66 & 104.00 & 127.40 & 2.93 & 53.43 & 49.50 & 2.96 & 8.82 & 46.66 \\
\hline 29 & DHS-29 & 42.33 & 102.33 & 75.33 & 3.03 & 43.20 & 49.66 & 2.73 & 6.96 & 46.00 \\
\hline 30 & DHS-30 & 41.33 & 106.00 & 77.90 & 2.63 & 47.40 & 51.20 & 2.96 & 8.50 & 44.86 \\
\hline 31 & DHS-31 & 46.33 & 108.33 & 105.96 & 2.40 & 36.93 & 43.06 & 3.00 & 6.07 & 46.10 \\
\hline 32 & DHS-32 & 47.00 & 105.33 & 95.50 & 2.63 & 47.50 & 49.16 & 2.90 & 7.92 & 45.86 \\
\hline 33 & DHS-33 & 43.00 & 103.00 & 117.43 & 3.33 & 47.46 & 56.60 & 3.00 & 9.57 & 50.20 \\
\hline 34 & DHS-34 & 41.33 & 109.66 & 94.90 & 2.36 & 40.96 & 49.40 & 3.16 & 7.70 & 46.60 \\
\hline 35 & DHS-35 & 48.00 & 105.00 & 101.70 & 2.80 & 50.63 & 54.76 & 3.16 & 9.67 & 46.33 \\
\hline 36 & DHS-36 & 42.66 & 104.00 & 165.40 & 3.36 & 52.13 & 50.46 & 3.06 & 8.93 & 45.66 \\
\hline 37 & DHS-37 & 41.00 & 101.66 & 91.06 & 3.40 & 50.66 & 46.10 & 2.96 & 8.63 & 49.40 \\
\hline 38 & DHS-38 & 44.66 & 105.33 & 95.90 & 2.43 & 40.96 & 54.20 & 2.90 & 7.28 & 51.06 \\
\hline 39 & DHS-39 & 48.00 & 102.66 & 97.93 & 2.23 & 38.53 & 50.50 & 2.93 & 6.86 & 47.23 \\
\hline 40 & DHS-40 & 43.00 & 104.00 & 94.33 & 2.26 & 39.26 & 50.10 & 3.10 & 7.02 & 47.00 \\
\hline 41 & JLT-408 & 43.00 & 105.00 & 121.43 & 2.96 & 56.23 & 51.33 & 3.13 & 8.16 & 48.56 \\
\hline \multirow[t]{5}{*}{42} & JLT-7 & 44.00 & 106.00 & 127.26 & 3.10 & 60.10 & 50.66 & 3.10 & 8.11 & 49.40 \\
\hline & G Mean & 44.79 & 104.06 & 106.52 & 2.63 & 44.13 & 49.36 & 2.98 & 7.61 & 47.39 \\
\hline & S.E ( \pm$)$ & 0.869 & 1.076 & 3.465 & 0.169 & 2.04 & 1.95 & 0.089 & 0.377 & 2.287 \\
\hline & C.D at $5 \%$ & 2.445 & 3.029 & 9.749 & 0.476 & 5.739 & 5.486 & 0.250 & 1.060 & 6.435 \\
\hline & C.V. (\%) & 3.36 & 1.79 & 5.63 & 11.12 & 8.00 & 9.84 & 5.16 & 8.57 & 8.36 \\
\hline
\end{tabular}


Table.2 Analysis of variance for different characters in sesamum

\begin{tabular}{|c|l|c|c|c|}
\hline \multirow{2}{*}{$\begin{array}{c}\text { Sr. } \\
\text { No. }\end{array}$} & \multicolumn{1}{|c|}{ Characters } & \multicolumn{3}{c|}{ Mean sum of square } \\
\hline $\mathbf{1}$ & Days to 50\% flowering & Replication & Genotype & Error \\
\hline $\mathbf{2}$ & Days to maturity & 6.746 & $17.202^{* *}$ & 2.266 \\
\hline $\mathbf{3}$ & Plant height at maturity $(\mathrm{cm})$ & 4.055 & $2240.86^{* *}$ & 36.025 \\
\hline $\mathbf{4}$ & Number of branches per plant & 0.517 & $0.541^{* *}$ & 0.086 \\
\hline $\mathbf{5}$ & Number of capsules per plant & $15.827 *$ & $191.632^{* *}$ & 12.485 \\
\hline $\mathbf{6}$ & Number of seeds per capsule & 18.448 & $29.926^{*}$ & 11.407 \\
\hline $\mathbf{7}$ & 1000 seed weight (g) & 0.081 & $0.097 * *$ & 0.023 \\
\hline $\mathbf{8}$ & Seed yield per plant $(\mathrm{g})$ & 0.447 & $4.090^{* *}$ & 0.426 \\
\hline $\mathbf{9}$ & Oil content $(\%)$ & 20.430 & $34.387 *$ & 15.699 \\
\hline
\end{tabular}

$*, * *$ Indicates significance at $5 \%$ and $1 \%$ level, respectively

Table.3 Grouping of forty-two Sesamum genotypes into different clusters

\begin{tabular}{|c|c|c|c|}
\hline $\begin{array}{l}\text { Sr. } \\
\text { No. }\end{array}$ & Cluster & $\begin{array}{l}\text { No. of } \\
\text { genotypes }\end{array}$ & Name of genotypes \\
\hline 1 & I & 10 & $\begin{array}{l}\text { JLT-408, JLT-7, DHS-28, DHS-23, DHS-33, DHS-27, DHS-4, } \\
\text { DHS-35, DHS-9, DHS-18. }\end{array}$ \\
\hline 2 & II & 16 & $\begin{array}{l}\text { DHS-24, DHS-32, DHS-2, DHS-38, DHS-21, DHS-40, DHS- } \\
\text { 39, DHS-22, DHS-26, DHS-13, DHS-31, DHS-7, DHS-1, } \\
\text { DHS-3, DHS-15, DHS-14. }\end{array}$ \\
\hline 3 & III & 1 & DHS-12 \\
\hline 4 & IV & 1 & DHS-17 \\
\hline 5 & V & 5 & DHS-11, DHS-29, DHS-30, DHS-37, DHS-25. \\
\hline 6 & VI & 1 & DHS-34 \\
\hline 7 & VII & 6 & DHS-16, DHS-36, DHS-19, DHS-5, DHS-20, DHS-6. \\
\hline 8 & VIII & 1 & DHS-10 \\
\hline 9 & IX & 1 & DHS-8 \\
\hline
\end{tabular}

Table.4 Average intra and inter-cluster distance ( $\mathrm{D}^{2}$ values) for nine characters in sesamum

\begin{tabular}{|c|c|c|c|c|c|c|c|c|c|}
\hline Clusters & I & II & III & IV & V & VI & VII & VIII & IX \\
\hline I & $\underline{2.83}$ & 4.58 & 6.84 & 5.58 & 5.53 & 4.72 & 5.84 & 7.25 & 3.45 \\
\hline II & & $\underline{2.94}$ & 3.67 & 3.55 & 4.11 & 3.66 & 7.60 & 4.22 & 3.89 \\
\hline III & & & $\underline{0.00}$ & 3.10 & 4.15 & 4.68 & 9.70 & 3.11 & 5.76 \\
\hline IV & & & & $\underline{0.00}$ & 3.19 & 3.64 & 8.74 & 4.28 & 5.14 \\
\hline V & & & & & $\underline{3.15}$ & 3.92 & 9.47 & 5.37 & 5.51 \\
\hline VI & & & & & & $\underline{0.00}$ & 8.17 & 5.06 & 4.91 \\
\hline VII & & & & & & & $\underline{3.75}$ & 9.71 & 4.96 \\
\hline VIII & & & & & & & & $\underline{0.00}$ & 6.26 \\
\hline IX & & & & & & & & & $\underline{0.00}$ \\
\hline
\end{tabular}

(Underlined figures indicate intra-cluster $\mathrm{D}^{2}$ values) 
Int.J.Curr.Microbiol.App.Sci (2020) 9(12): 884-893

Table.5 Cluster means of forty-two genotypes for nine characters in Sesamum

\begin{tabular}{|c|c|c|c|c|c|c|c|c|c|c|c|}
\hline \multirow[t]{2}{*}{ Sr. No. } & \multirow[t]{2}{*}{ Characters } & \multicolumn{9}{|c|}{ Clusters } & \multirow[t]{2}{*}{ Average } \\
\hline & & I & II & III & IV & $\mathbf{V}$ & VI & VII & VIII & IX & \\
\hline 1 & $\begin{array}{l}\text { Days to } 50 \% \\
\text { flowering }\end{array}$ & 45.20 & 45.81 & 45.33 & 40.67 & 42.80 & 41.33 & 43.89 & 47.33 & 44.33 & 44.07 \\
\hline 2 & $\begin{array}{l}\text { Days to } \\
\text { maturity }\end{array}$ & 104.67 & 104.23 & 103.33 & 101.00 & 103.33 & 109.67 & 103.28 & 102.67 & 103.33 & 103.94 \\
\hline 3 & $\begin{array}{l}\text { Plant height at } \\
\text { maturity }(\mathrm{cm})\end{array}$ & 115.07 & 94.23 & 74.17 & 83.50 & 75.51 & 94.90 & 161.28 & 84.47 & 123.87 & 100.77 \\
\hline 4 & $\begin{array}{l}\text { No. of } \\
\text { branches per } \\
\text { plant }\end{array}$ & 3.01 & 2.38 & 2.00 & 2.00 & 2.83 & 2.37 & 2.86 & 2.00 & 3.07 & 2.50 \\
\hline 5 & $\begin{array}{l}\text { No. of } \\
\text { capsules per } \\
\text { plant }\end{array}$ & 51.74 & 40.69 & 33.43 & 36.97 & 45.23 & 40.97 & 46.07 & 23.63 & 47.37 & 40.67 \\
\hline 6 & $\begin{array}{l}\text { No. of seeds } \\
\text { per capsule }\end{array}$ & 51.60 & 48.55 & 48.73 & 48.60 & 47.78 & 49.40 & 50.81 & 44.33 & 45.73 & 48.39 \\
\hline 7 & $\begin{array}{l}1000 \text { seed } \\
\text { weight }(\mathrm{g})\end{array}$ & 3.11 & 2.97 & 2.63 & 2.73 & 2.91 & 3.17 & 2.96 & 3.20 & 2.93 & 2.95 \\
\hline 8 & $\begin{array}{l}\text { Oil content } \\
(\%)\end{array}$ & 48.78 & 47.28 & 46.00 & 47.63 & 47.57 & 46.60 & 45.64 & 44.30 & 50.17 & 47.10 \\
\hline 9 & $\begin{array}{l}\text { Seed yield per } \\
\text { plant }(\mathrm{g})\end{array}$ & 8.82 & 6.92 & 5.54 & 8.20 & 7.66 & 7.70 & 8.08 & 6.06 & 6.56 & 7.28 \\
\hline
\end{tabular}

Table.6 Relative per cent contribution of different characters towards total genetic divergence in sesamum

\begin{tabular}{|c|l|c|c|}
\hline Sr. No. & \multicolumn{1}{|c|}{ Characters } & $\begin{array}{c}\text { Times ranked } \\
\text { 1st }\end{array}$ & $\begin{array}{c}\text { Per cent } \\
\text { contribution }\end{array}$ \\
\hline $\mathbf{1}$ & Days to 50\% flowering & 78 & 9.06 \\
\hline $\mathbf{2}$ & Days to maturity & 26 & 3.02 \\
\hline $\mathbf{3}$ & Plant height at maturity (cm) & 448 & 52.03 \\
\hline $\mathbf{4}$ & Number of branches per plant & 48 & 5.57 \\
\hline $\mathbf{5}$ & Number of capsules per plant & 119 & 13.82 \\
\hline $\mathbf{6}$ & Number of seeds per capsule & 17 & 1.97 \\
\hline $\mathbf{7}$ & 1000 seed weight (g) & 17 & 1.97 \\
\hline $\mathbf{8}$ & Seed yield per plant (g) & 16 & 1.86 \\
\hline $\mathbf{9}$ & Oil content (\%) & 92 & 10.69 \\
\hline & TOTAL & $\mathbf{8 6 1}$ & $\mathbf{1 0 0}$ \\
\hline
\end{tabular}

Table.7 Divergence classes

\begin{tabular}{|l|c|r|r|r|r|}
\hline & DC 4 & DC 3 & DC 2 & & DC 1 \\
\hline$\downarrow$ & $\downarrow$ & $\downarrow$ & & $\downarrow$ & $\downarrow$ \\
\hline $\mathrm{X}$ & $\mathrm{m}-\mathrm{s}$ & $\mathrm{m}$ & & $\mathrm{m}+\mathrm{s}$ & $\mathrm{Y}$ \\
\hline$(2.83)$ & $(3.48)$ & $(4.57)$ & & $(5.66)$ & $(9.70)$ \\
\hline
\end{tabular}


Table.8 Distribution of different clusters combinations into four divergence classes based on $\mathrm{D}^{2}$ values between them. (Cluster combinations)

\begin{tabular}{|c|c|}
\hline & $\mathrm{Y}(9.70)$ \\
\hline \multirow[t]{2}{*}{ DC 1} & $\begin{array}{l}\text { (I,III), (I,VII), (I,VIII), (II,VII), (III,VII), (III,IX), (IV,VII), } \\
\text { (V,VII),(VI,VII), (VII,VIII), (VIII,IX) }\end{array}$ \\
\hline & $\mathrm{m}+\mathrm{s}(5.66)$ \\
\hline \multirow[t]{2}{*}{ DC 2} & $\begin{array}{l}\text { (I,II), (I,IV), (I,V), (III,VI),(IV,IX), (V,VIII), (VI,IX), (VI,VIII), (VI,IX), } \\
\text { (VII,IX) }\end{array}$ \\
\hline & $\mathrm{m}(4.57)$ \\
\hline \multirow[t]{2}{*}{ DC 3} & $\begin{array}{l}\text { (I,IX), (II,III), (II,IV), (II,V), (II,VI), (II,VIII), (II,IX), (III,V), (IV,VI), (IV,VIII), } \\
\text { (V,VI), VII,VII) }\end{array}$ \\
\hline & m-s (3.48) \\
\hline \multirow[t]{2}{*}{ DC 4} & $(\mathrm{I}, \mathrm{I}),(\mathrm{II}, \mathrm{II}),(\mathrm{III}, \mathrm{IV}),(\mathrm{III}, \mathrm{VII}),(\mathrm{IV}, \mathrm{V}),(\mathrm{V}, \mathrm{V})$ \\
\hline & $X(2.83)$ \\
\hline
\end{tabular}

Table.9 Characters improvement on the basis of source clusters

\begin{tabular}{|c|l|c|}
\hline Sr. No. & \multicolumn{1}{|c|}{ Characters } & Source Clusters \\
\hline 1 & Days to 50 \% flowering (Early) & IV,VI,V \\
\hline 2 & Days to maturity (Early) & IV,VIII,VII,III,V \\
\hline 3 & Plant height at maturity (cm) (Tall and Dwarf) & VII,IX,I \\
\hline 4 & Number of branches per plant (Maximum) & IX,I,VII,V \\
\hline 5 & Number of capsules per plant (Maximum) & I,IX,VII,V \\
\hline 6 & Number of seeds per capsule (Maximum) & I,VII,VI,III,II \\
\hline 7 & 1000 seed weight (Maximum) & VIII,VI,I,II \\
\hline 8 & Oil content (Maximum) & IX,I,IV,V,II \\
\hline 9 & Seed yield per plant (Maximum) & I,IV,VII,V \\
\hline
\end{tabular}

Genetic divergence which is due to genetic factors is the basis for heritable improvement. The plant breeders have always therefore been fascinated by great amount of diversity in crop plants. The precise information about the genetic divergence therefore, is crucial for effective breeding programme.
The genetically diverse parents are known to produce high heterotic effects and consequently gives desirable recombinants in the breeding material or wide spectrum of transgressive segregants in segregating generations. Hence, the systemic management 
of plant genetic resources is very important to augment productivity of sesamum.

The Mahalanobis $\mathrm{D}^{2}$ statistics are computed for all possible pair of forty-two genotypes in order to assess the genetic diversity present among the genotypes under study. Wilk's criterion showed the significant differences between the genotypes for the pooled effect of nine characters (Wilk's criteria $\mathrm{X}^{2}=13241.45$ at 390 df.) hence further analysis was carried out to calculate the $\mathrm{D}^{2}$ values. The calculated $\mathrm{D}^{2}$ values ranged from 2.015 (DHS-32) to 289.463 (DHS-7) based upon the observation of nine characters.

In the present study, the genotypes belonging to the same geographical region or same location fall into different clusters and accordingly these clusters were separated by high genetic distances.

Hays and Johansson (1939) and East and Hays (1942) obtained maximum heterosis from crosses between diverse parent than those between closely related ones. Bhatt (1970) advocated the use of multivariate analysis for the selection of parents. He also stated that statistical distance of all possible cluster combination may be considered arbitrarily as a guideline and suggested that it would be logical to effect crosses between genotypes belonging to cluster separated by high estimated statistical distance.

Grouping of cluster pairs into the divergence class (DC) are presented in Table 8. In the light of discussion, initial choice of parents should be made from the cluster combinations falling in the divergence classes DC2 and DC3. While crossing among the genotypes of a cluster, the per se performance of the genotypes for different traits such as earliness (days to $50 \%$ flowering and days to maturity), plant height at maturity, number of branches per plant, number of capsules per plant, number of seeds per capsule, seed yield per plant, 1000 seed weight and oil content etc. should be taken into account. So, that desirable transgressivie segregants would be obtained after hybridization.

The cluster means presented in Table 6 considering, the cluster means or the various clusters which can provide the desired parents for hybridization programmes for improvement in the characters shown against them are listed (Table 7 and 9).

Keeping in view all the above aspects, the following genotypes in the present studies, deserve to be considered as potent parents for future crossing programme for improvement of seed yield and yield contributing characters.

$\begin{array}{llll}1 & \text { DHS-5 } & 6 & \text { DHS-17 } \\ 2 & \text { DHS-11 } & 7 & \text { DHS-33 } \\ 3 & \text { DHS-18 } & 8 & \text { DHS-35 } \\ 4 & \text { DHS-22 } & 9 & \text { DHS-38 } \\ 5 & \text { DHS-28 } & 10 & \text { DHS-36 }\end{array}$

Considering the inter-cluster distance, cluster means, per se performance of genotypes and divergence class, the above genotypes may be utilized in future breeding programme for creating maximum spectrum of variability for different yield contributing characters which will facilitate to develop superior genotypes with respect to more than one characters and also possible to improve more than one character simultaneously.

Similar studies was carried out by Bandila et al., (2011), Ahadu (2012), Narayanan and Murugan (2013), Chandra (2014), Hira et al., (2014), Hemalata and Saundarya (2015), Iqbal et al., (2016), Yirga Belay Kindeya (2017), Saundharya et al., (2017). 


\section{References}

Ahadu, M. 2012. Phenotypic variability, divergence analysis and heritability of characters in sesame (Sesamum indicum L.) genotypes. Journal of Nature and Science Research, 10(10):117-121.

Arunachalam, V. 1981. Genetic distances in plant breeding. Indian Journal of Genetics, 41: 226-236.

Arunachalam, V. and A. Bandopadhay. 1984. Limits to genetic divergence for occurrence of heterosis experimental evidence from crop plants. Indian Journal of Genetics, 44 (3): 548-554.

Bandila, S. Chanta, A. Natrajan, S. and Subramanian. 2011. Determination of genetic variation in Indian sesame genotypes for morphological traits. Journal of Research in Agriculture Science. 7(2): 88-89.

Bhatt, G. M. 1970. Multivariate analysis approach to selection of parents for hybridization, assuming of yield improvement in self-pollinated crops. Australian Journal of Agriculture Research, 21: 1-17.

Chandra, M. 2014. Variability and genetic divergence in sesame (Sesamum indicum L.). International Journal of applied biology and pharmaceutical technology, 5 (3):0976-4550.

East, E. M. and Hayes, H. K. 1942. Heterozygosis in evolution and plant Breeding U.S.D.A. Bureau of plant Industry Bulletin 243: 1-58

Falconer, D. S. 1989. An introduction to Quantitative Genetics. Longman, New York.

Hays, H. K. and Johnson, I. J. 1939. The breeding of self-improved lines of corn. Journal of American Society of Agronomy, 31: 710-724.

Hemalatha, V., and Soundharya, B. (2015), Genetic diversity studies in sesame
(Sesamum indicum L.) genotypes. Thesis submitted to Prof. Jayashankar Telangana State Agricultural University. Hyderabad.

Hika, G.; Gelata, N. and Jaleta, Z. 2014. Correlation and Divergence analysis for phenotypic traits in sesame (Sasamum indicum L.) Genotypes. Science Technology Arts Research Journal, 3(4): 01-09.

Iqbal, A., Akhtar, R., Begun, T. and Dasgupta T. 2016. Genetic estimates and diversity study in Sesame (Sesamum indicum L.). Journal of Agriculture and Variability Science, 9:2319-2372.

Kindeya, Y. B. 2017. Correlation and cluster analysis of white seeded sesame (Sesamum indicum L.) genotypes oil yield in northern Ethiopia. African Journal of Agricultural Research, 12(12): 970-978.

Mahalanobis, P.C., 1936. On the generalized distance in statistics. Proceedings of the National Academy of Science, 2:55-79.

Narayanan, R. and Murugan, S. 2013. Studies on genetic diversity and heritability in sesamum. International Journal of current Agriculture Research, 2 (11):52-55.

Panse, V. G. and Sukhatmate, P. V. 1995. Statistical method for Agricultural worker. ICAR, New Delhi $4^{\text {th }}$ Edn. pp. 145-150.

Rao, C. R. 1952. Advanced Statistical Methods in Biometrical Research. John Willey and Sons, New York.

Soundharya, B., Hemlatha, V., Shobha Rani T and Edukondalu, B. 2017. Genetic divergence studies in Sesame (Sesamum indicum L.) genotypes. International Journal of Current Microbiology and Applied Sciences, 6(9), 2615-2619. 


\section{How to cite this article:}

Rohini Y. Patil, K. K. Barhate, B. A. Tagad and Girase, V. S. 2020. Genetic Diversity Studies in Sesamum. Int.J.Curr.Microbiol.App.Sci. 9(12): 884-893.

doi: https://doi.org/10.20546/ijcmas.2020.912.106 